\title{
Design of de-coupler for an interacting tanks system
}

\author{
Parag Joshi \\ Department Of Chemical Engineering, Jaipur National University, India
}

\begin{abstract}
The dynamic behaviour of two tank interacting system was studied by the introduction of a step change in the manipulated variable (flow rate) and measuring the controlled variable by development of the suitable mathematical model of the system. This paper describes how the effect of the interaction of this interacting system is minimized by the design of the suitable de-coupler for the system and also includes the analysis of the interacting loops between the controlled variable that is liquid level of both tank and manipulated variable that is inlet flow rate. Generally in two tank interacting system there is no inlet flow rate for tank two, but this paper simplifies the industrial interaction problem where different tanks has different inlet and this is done by the help of relative gain array method. The result exemplifies that the gain of each loop is reduced approximately half when the opposite loop is closed and the gain of other loop changes the sign when the opposite loop is closed. Thus the decoupling is the most appropriate method for achieving minimal interaction and is most efficient method.
\end{abstract}

Keywords: Controlled variable, De-coupler, Interacting loops, Interacting tanks, Manipulated variables.

\section{Introduction}

Most of the chemical industries have a cascade of tanks for storage and also for the chemical processes. Generally the have different inlet flow streams but are inter connected by a resistance line makes the whole as a interacting tanks system series. The tanks have their own manipulated variable to maintain the level inside the tanks and that is flow rate and this manipulated variable controls the liquid level by continuous regulation of themselves manually for a large number of tanks and need high investment for labours. Thus we are temptated towards the design of a control system which makes an ease to control the controlled variable or in general the liquid level.

Such a system with multi input and multi output variables are often referred as MIMO systems and it contains several industrial systems like heat exchangers, chemical reactors and also the distillation column etc. Systems with single input and single output variables are SISO systems. It is easy to perform an operation or process with SISO system in comparison of MIMO system. The reason behind this complication is the interaction between the input and output variables.

The interacting system are so called because each input to the system affects more than one output and this sometimes known as loading or interaction otherwise the system in non-interacting. Here we are discussing about the control system with two interacting tanks system and the field of interest is to reduce the interaction by designing a suitable control system or a new element what is called as de-coupler. The purpose of the decoupler is to reduces the interaction effect between two loops and thus render two non interacting control loops. We can say that the goal of the de-coupler is to decouple the two interacted tanks or to eliminate the complicated loop interaction that results a system in which and change in the process variable does not influences the another process variable.

The paper provides a realistic and experimental result for minimizing the industrial problem of interaction by designing a suitable de-coupler.

The study of the system is done by one of the most efficient and reliable method, that is relative gain array method.

1.1 RGA- Relative gain method is introduced by Bristol (1966), and is not only a valuable method for the selection of manipulated controlled variable pairings, but also predict the behaviour of control responses. RGA will be constructed for a system represented by p-canonical structure. RGA indicates how the input should be coupled with the output to form loops with a minimal interaction. It gives the condition of two different tanks system and four loop of two tanks system within a square matrix with the relative gains as components. A process with any controlled outputs and $\mathrm{N}$ manipulated variables, there are $\mathrm{N}$ ! different ways to form the controlled loops. The RGA provides exactly such a methodology, whereby we select pairs of input and output variables in order to minimize the amount of interaction among the resulting loops.

The paper deals with the study of the dynamic behaviour of the two tanks interacting system with the RGA method experimentally as well as the theoretical dynamic behaviour and provides the condition for choosing appropriate loop that have a minimal interaction. 


\subsection{Notations}

\section{Theory}

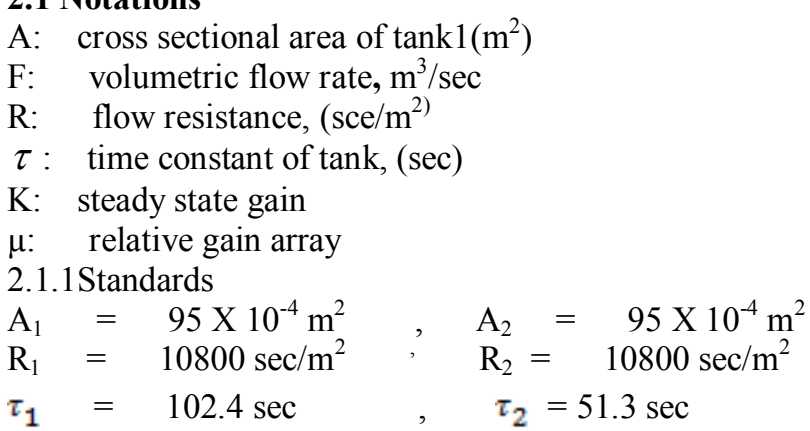

\subsection{Mathematical model}

The two tank interacting system is defined mathematically by a mathematical model that is derived on the basis of the assumption and that are:-

- The flow resistance is linear with the linear liquid level in the tanks.

- The tanks are of equal and uniform cross sectional area.

- The density of water remains constant or the fluid is incompressible.

A general schematic of two tank interacting system

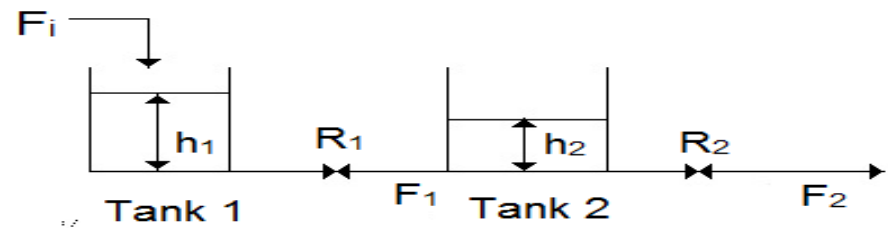

Fig1:-Interacting tanks system.

Applying the general material balance in both of the tanks

Accumulation $=$ flow rate in - flow rate out

For $1^{\text {st }}$ tank:

For $2^{\text {nd }}$ tank:

$$
A_{1} \frac{d h_{1}}{d t}=F_{i}-F_{1}------(i)
$$

By using assumptions:

$$
A_{2} \frac{d h_{2}}{d t}=F_{1}-F_{2}------(i i)
$$

$$
F_{1}=\frac{\left(h_{1}-h_{2}\right)}{R_{1}} \quad \text { and } \quad F_{2}=\frac{h_{2}}{R_{2}}
$$

then equations (i) and (ii) become:

$$
\begin{aligned}
& A_{1} R_{1} \frac{d h_{1}}{d t}+h_{1}-h_{2}=R_{1} F_{1}----(i i i) \\
& A_{2} R_{2} \frac{d h_{2}}{d t}+\left(1+\frac{R_{2}}{R_{1}}\right) h_{2}-\frac{R_{2}}{R_{1}} h_{1}=0--(i v) \\
& h_{1, s}-h_{2, s}=R_{1} F_{i, s}----(V) \\
& \left(1+\frac{R_{2}}{R_{1}}\right) h_{2, s}-\frac{R_{2}}{R_{1}} h_{1, s}=0----(v i)
\end{aligned}
$$

then steady state equivalents of equations (iii) and (iv) are subtracting equation (v) from (iii) and (vi) from (v) and then introducing deviation variables we get:

$$
A_{1} R_{1} \frac{d h^{\prime}}{d t}+h_{1}^{\prime}-h_{2}^{\prime}=R_{1} F^{\prime}{ }_{i}----(v i i)
$$


Where:

$$
A_{2} R_{2} \frac{d h_{2}^{\prime}}{d t}+\left(1+\frac{R_{2}}{R_{1}}\right) h_{2}^{\prime}-\frac{R_{2}}{R_{1}} h_{1}^{\prime}=0 . .(v i i i)
$$

$h_{1}^{\prime}=h_{1}-h_{1, s}$

$h_{2}^{\prime}=h_{2}-h_{2, s}$

$F_{i}^{\prime}=F_{i}-F_{i, s}$

taking the Laplace transform of equations (vii) and (viii) and find:

${\overline{h^{\prime}}}_{1}(s)=\frac{\left(\tau_{p 2} R_{1}\right) s+\left(R_{1}+R_{2}\right)}{\tau_{p 1} \tau_{p 2} s^{2}+\left(\tau_{p 1}+\tau_{p 2}+A_{1} R_{2}\right) s+1}{\overline{F^{\prime}}}_{i}(s)--------($ viii $)$

And:

$\bar{h}_{2}^{\prime}(s)=\frac{R_{2}}{\tau_{p 1} \tau_{p 2} s^{2}+\left(\tau_{p 1}+\tau_{p 2}+A_{1} R_{2}\right) s+1} \bar{F}_{i}(s)----------(i x)$

\subsection{Loop interaction}

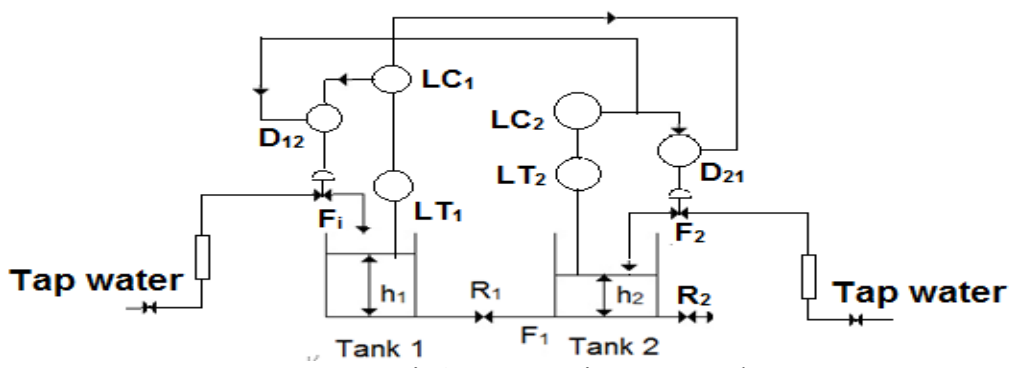

Fig2:-Interacting two tanks system

Let us consider a two tanks interacting system as shown in figure 2 having two manipulated variables $F_{i}$ and $F_{2}$. Now we need to find the gain of each manipulated variable on each of the controlled variable. The four open loop system is shown in the figure 3 below

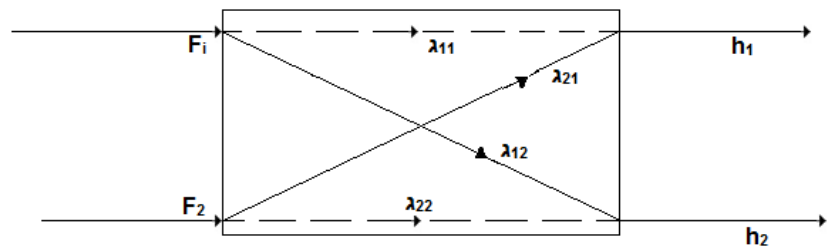

Fig3:- Schematic of interaction for controlled variable and manipulated variable and given as

$$
\begin{array}{cc}
\lambda_{11}=\left[\frac{\Delta h_{1}}{\Delta F_{i}}\right]_{F_{2}} & \lambda_{12}=\left[\frac{\Delta h_{1}}{\Delta F_{2}}\right]_{F_{i}}---(X) \\
\lambda_{21}=\left[\frac{\Delta h_{2}}{\Delta F_{i}}\right]_{F_{2}} & \lambda_{22}=\left[\frac{\Delta h_{2}}{\Delta F_{2}}\right]_{F_{i}}--(x i)
\end{array}
$$

where $\mu_{\mathrm{ij}}$ is the gain of $\mathrm{i}^{\mathrm{th}}$ controlled variable relating to the $\mathrm{j}^{\mathrm{th}}$ manipulated variable. The major advantage of RGA is that it requires only the steady state process parameters that are steady state gains. Thus

$$
\begin{aligned}
& \Delta h_{1}=\lambda_{11} \Delta F_{i}+\lambda_{12} \Delta F_{2}----(x i i) \\
& \Delta h_{2}=\lambda_{21} \Delta F_{i}+\lambda_{22} \Delta F_{2}----(x i i i)
\end{aligned}
$$

Now we need to introduce feedback system which connects $F_{2}$ with $h_{2}$, in order to determine the gain which connects $F_{i}$ with $h_{1}$ According to figure 3, we get four closed steady state loops and hence four steady state gains is to be calculated from the four open loop gains. Thus the resulting formulae are:-

$$
\begin{array}{ll}
\bar{\lambda}_{11}=\frac{\lambda_{11} \lambda_{22}-\lambda_{12} \lambda_{21}}{\lambda_{22}}, & \bar{\lambda}_{12}=\frac{\lambda_{11} \lambda_{22}-\lambda_{12} \lambda_{21}}{-\lambda_{21}} \\
\bar{\lambda}_{21}=\frac{\lambda_{11} \lambda_{22}-\lambda_{12} \lambda_{21}}{-\lambda_{12}}, & \bar{\lambda}_{22}=\frac{\lambda_{11} \lambda_{22}-\lambda_{12} \lambda_{21}}{\lambda_{11}}
\end{array}
$$


According to the definition,

Thus,

$$
\mu_{i j}=\frac{\lambda_{i j}}{\lambda_{i j}}----(x v)
$$

$$
\begin{aligned}
& \mu_{11}=\frac{\lambda_{11} \lambda_{22}}{\lambda_{11} \lambda_{22}-\lambda_{12} \lambda_{21}}, \mu_{12}=\frac{-\lambda_{12} \lambda_{21}}{\lambda_{11} \lambda_{22}-\lambda_{12} \lambda_{21}} \\
& \mu_{21}=\frac{-\lambda_{12} \lambda_{21}}{\lambda_{11} \lambda_{22}-\lambda_{12} \lambda_{21}}, \mu_{22}=\frac{\lambda_{11} \lambda_{22}}{\lambda_{11} \lambda_{22}-\lambda_{12} \lambda_{21}}
\end{aligned}
$$

\subsection{Decoupling of interacting loops}

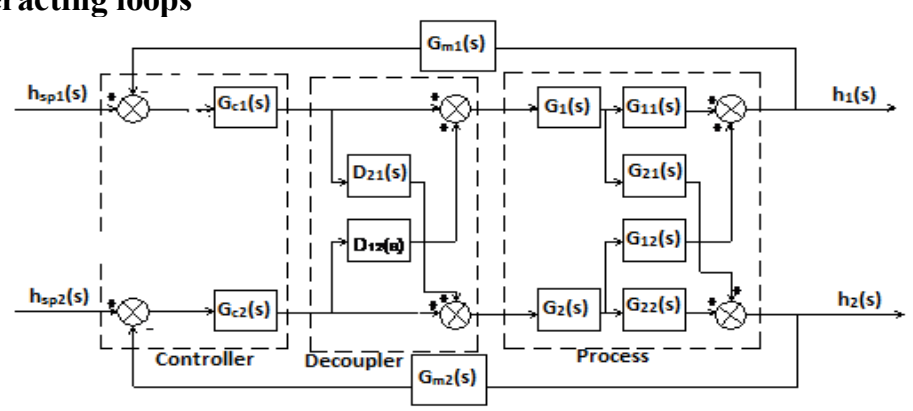

Fig 4:- block diagram of interacting tanks system with de-coupler

Decoupling does change to each loop what the other loops were going to do anyway, this is one of the important characteristic of de-coupler. The de-coupler control system works as feedback system for tank 1 and the same as feed forward for the tank 2. In order to reduce this interaction between two tanks, two de-coupler blocks having transfer functions $\mathrm{D}_{12}(\mathrm{~s})$ and $\mathrm{D}_{21}(\mathrm{~s})$ are installed in the system where de-coupler $\mathrm{D}_{12}(\mathrm{~s})$ cancels the effect of manipulated variable $\mathrm{F}_{2}(\mathrm{~s})$ on controlled variable $\mathrm{h}_{1}(\mathrm{~s})$ and $\mathrm{D}_{21}(\mathrm{~s})$ cancels the effect of manipulated variable $\mathrm{F}_{\mathrm{i}}(\mathrm{s})$ on controlled variable $\mathrm{h}_{2}(\mathrm{~s})$. Thus we get the design formulae for the de-couplers are:-

$$
\begin{gathered}
D_{12}(s)=-\frac{G_{2}(s) G_{12}(s)}{G_{1}(s) G_{11}(s)}---(\text { xvii }) \\
D_{21}(s)=-\frac{G_{1}(s) G_{21}(s)}{G_{2}(s) G_{22}(s)}----(\text { xviii })
\end{gathered}
$$

\subsection{Experimental Work}

\section{Materials and Methods}

\subsubsection{Description of Equipment}

- Interacting two tanks - The interacting two tanks consists of two vessels arranging cascade having each of diameter $\mathrm{m}$, and hence the area $\mathrm{m}^{2}$ respectively. Small narrow pipe with valve is connected between the two tanks.

- Water is supplied through stainless steel float rotameter having range 0-60 LPH with temperature condition of water at $25^{\circ} \mathrm{C}$. The Rotameter has maximum capacity of $60 \mathrm{LPH}$.

- The control system consist of PID controller, control valve and pressure transmitter.

- Resistances are provided to control the inlet flow of the constant manipulated variable of the opposite tank.

- Rotameters are employed in the inlet line of tank two and same as for tank 1 while dealing with the manipulated variable of tank 2 .

\subsubsection{Experimental procedure}

The inlet streams were given to the tanks and the valve in the inlet streams were adjusted to provide a suitable reading on the rotameter. After sometimes the liquid levels in two tanks are in steady state and system was left for stabilization. Then the following step were performed:-

$>$ The inlet flow rate of the tank $1\left(\mathrm{~F}_{\mathrm{i}}\right)$ was stepped up from $14 \mathrm{LPH}$ to $18 \mathrm{LPH}$ with constant inlet flow rate of tank $2\left(\mathrm{~F}_{2}\right)$ at $10 \mathrm{LPH}$ by using the valve and the liquid levels in both tanks were recorded with respect to time.

$>$ The inlet flow rate of the tank $2\left(\mathrm{~F}_{2}\right)$ was stepped up from $10 \mathrm{LPH}$ to $14 \mathrm{LPH}$ with constant inlet flow rate of tank $1\left(\mathrm{~F}_{\mathrm{i}}\right)$ at $14 \mathrm{LPH}$ by using the valve and the liquid levels in both tanks were recorded with respect to time. 
The inlet flow rate of the tank $1\left(\mathrm{~F}_{\mathrm{i}}\right)$ was stepped down from $14 \mathrm{LPH}$ to $10 \mathrm{LPH}$ with constant inlet flow rate of tank $2\left(\mathrm{~F}_{2}\right)$ at $10 \mathrm{LPH}$ by using the valve and the liquid levels in both tanks were recorded with respect to time.

$>$ The inlet flow rate of the tank $2\left(\mathrm{~F}_{2}\right)$ was stepped down from $10 \mathrm{LPH}$ to $7 \mathrm{LPH}$ with constant inlet flow rate of tank $1\left(\mathrm{~F}_{\mathrm{i}}\right)$ at $14 \mathrm{LPH}$ by using the valve and the liquid levels in both tanks were recorded with respect to time.

\section{Results And Discussions}

Relative gain array $\mu_{\mathrm{ij}}$ is calculated from both experimental and theoretical final steady state condition with a step change in inlet flow rate of the two tanks. Theoretically it is found the relative gain as, each divides the gain exactly half while the other is closed for the loop 1 while the other is closed with a negative relative gain of -1 and for the tank itself the de-coupler works as feedback system and the gain of each loop is divided in half and for each feedback system for which the de-coupler acting has a steady state gain of 2 .

$$
\mu_{\text {Theoretical }}=\left[\begin{array}{lr}
2 & -1 \\
-1 & 2
\end{array}\right] \quad \mu_{\text {Experimetal }}=\left[\begin{array}{cr}
1.55 & -0.52 \\
-0.55 & 1.55
\end{array}\right]
$$

Here the experimental results satisfies the theoretical results and are reliable. The relative gain indicates that the gain loop changes sign when the other loop is closed. Obviously last condition was undesirable. it is because of the fact that the action of the controller depends on the, whether the loop is opened or closed.

The results give the design formula for the de-coupler design are given as

$$
D_{12}(s)=-\frac{0.5}{51.3 S+1} \quad, \quad D_{21}(s)=-\frac{2}{102.4 S+1}
$$

Thus we can say that both the de-couplers are simple gains. De-coupler $\mathrm{D}_{12}(\mathrm{~s})$ has a negative gain because its main intention is to keep the liquid level of tank $1\left(\mathrm{~h}_{1}\right)$, when the second inlet stream $\left(\mathrm{F}_{2}\right)$ changes. Similarly, Decoupler $\mathrm{D}_{12}(\mathrm{~s})$ has a positive gain because its main intention is to keep the liquid level of tank $2\left(\mathrm{~h}_{2}\right)$, when the first inlet stream $\left(\mathrm{F}_{1}\right)$ changes. The experimental study with the time of each tank is shown in the figures
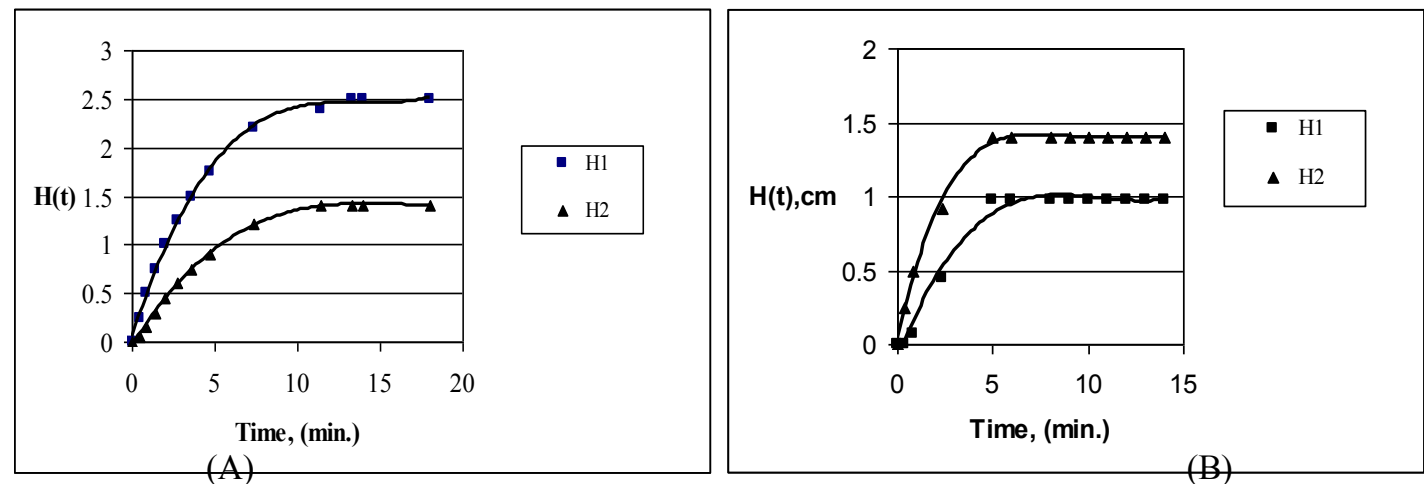

Experimental responses of liquid level to step change in inlet flow rate of the tanks when step change is stepped up , (A) show 14 to $10 \mathrm{LPH}$ for tank 1 and (B) for 10 to $14 \mathrm{LPH}$ for tank 2
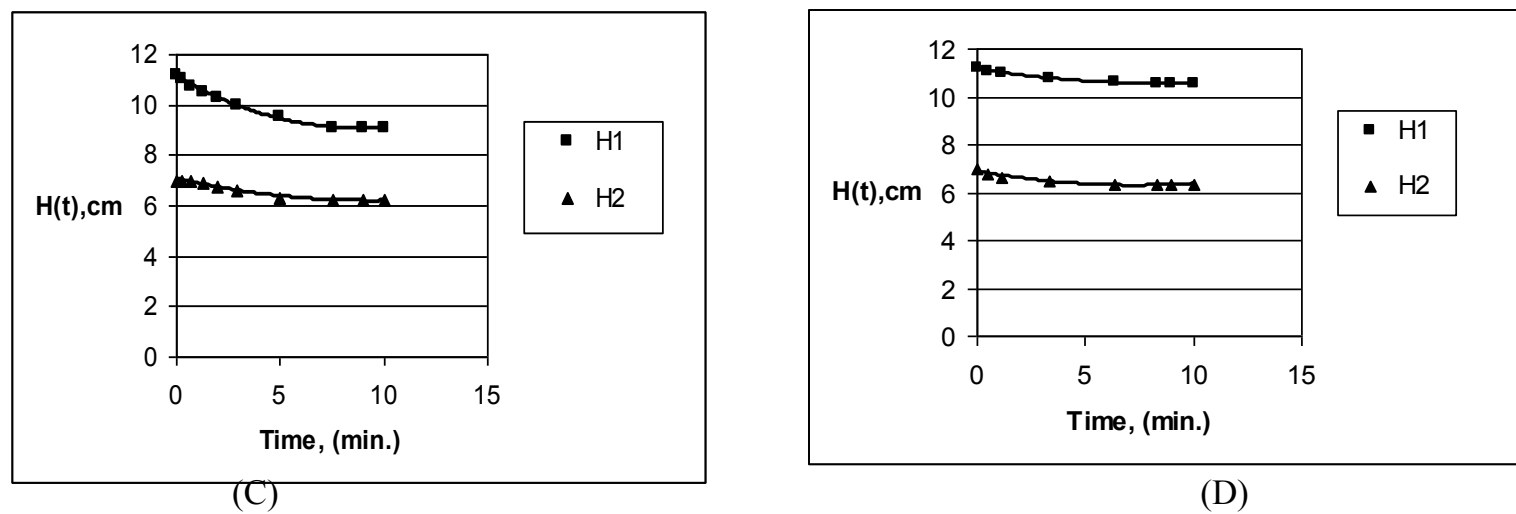

(D)

Experimental responses of liquid level to step change in inlet flow rate of the tanks when flow rate is stepped down, (C) show 14 to $10 \mathrm{LPH}$ for tank 1 and (D) shows 10 to $7 \mathrm{LPH}$ for tank 2 


\section{Conclusion}

Thus from the above experiments, the following conclusions are made

$>$ The de-couplers lessen the interaction of two interacting tanks system and the de-couplers have same effect as it has before.

$>$ Since the relative gain is It means that when the loop 1 is closed, the gain of loop 2 is cut into half.

$>$ Since the relative gain is it means that the action of controller depends on either the loop is open or close and it is undesirable one.

$>$ It also results that de-coupler can't totally cancels the interaction of the cascade but it can reduces the interaction much effectively. $\quad$ NOTE: Here the effect of the decoupling control system is studied and the outputs are measured to find the proper loop that provide minimal interaction not he whole description of the dynamic behaviour is shown by plotting the height with time until the height in both the tanks reached at steady state.

\section{Acknowledgement}

Highly acknowledged by the Department of Chemical Engineering, Jaipur National University for providing process dynamic and control lab and grateful for the support by providing lab for extra work after schedule of the department

\section{References}

[1] Zumberge J., Passino, K.M., A case study in Intelligent vs. Conventional Control for a process control Experiment, Journal of control Engineering practice, Vol. 6, No. 9, Page No. 1055-1075,1998.

[2] TriellWeiler J.O., Application of The Robust Performance Number for Quantification of the Operability of the Quadruple-Tank Process, Brazilian Journal of Chemical Engineering, Vol. 19, P Page No. 195-206.

[3] Esche S.K.,Tank Filling Of Liquid Level System, Stevens Institute of Technology, 2001,

[4] Stephanopoulos G., Chemical Process Control, An Introduction toTheory and Practice, Prentice- Hall India, Inc.,1984, Page No. 487-508.

[5] Luyben L. William Process Modeling, Simulation and Control for Chemical Engineers McGraw-Hill International Editions1996,page No. 573-584.

[6] Coughanowr R. Donald Process Systems Analysis and Control McGraw-Hill International Editions Inc. 1965 Chemical Engineering Series, Page No. 82-86.

[7] Edgar T. F. \& Rueda L. Process Dynamic and Control Laboratory Experiments Carried Out Over The Internet University of Texas, 2003.

[8] Ibrahim D. Microcontroller Based Applied Digital Control John Wiley \& Son Ltd., Page No. 269-282, 2006. 